\title{
Non-Uniform Drug Distribution Matrix System (NUDDMat) for Zero-Order Release of Drugs with Different Solubility
}

\section{Authors}

5 Matteo Cerea ${ }^{1}$, Anastasia Foppoli ${ }^{1,2}$, Luca Palugan $^{1}$, Alice Melocchi ${ }^{1}$, Lucia Zema $^{1}$, Alessandra Maroni ${ }^{1}$, Andrea Gazzaniga $^{1}$

${ }^{1}$ Università degli Studi di Milano

Dipartimento di Scienze Farmaceutiche

10 Sezione di Tecnologia e Legislazione Farmaceutiche "Maria Edvige Sangalli"

via G. Colombo 71

20133 Milano, Italy

${ }^{2}$ Corresponding author

15 Università degli Studi di Milano

Dipartimento di Scienze Farmaceutiche

Sezione di Tecnologia e Legislazione Farmaceutiche "Maria Edvige Sangalli"

via G. Colombo 71

20133 Milano, Italy

$20 \quad$ Tel +390250324654

email: anastasia.foppoli@unimi.it 


\title{
Keywords
}

Hydrophilic matrices, powder layering, gradient concentration, oral prolonged release, zero-order kinetics, tangential spray rotary fluid bed.

\begin{abstract}
A decrease in the drug release rate over time typically affects the performance of hydrophilic matrices for oral prolonged release. To address such an issue, a Non-Uniform Drug Distribution Matrix (NUDDMat) based on hypromellose was proposed and demonstrated to yield zero-order release. The system consisted of 5 overlaid layers, applied by powder layering, having drug concentration decreasing from the inside towards the outside of the matrix according to a descending staircase function. In the present study, manufacturing and performance of the described delivery platform were evaluated using drug tracers having different water solubility. Lansoprazole, acetaminophen and losartan potassium were selected as slightly (SST), moderately (MST) and highly (HST) soluble tracers. By halving the thickness of the external layer, which contained no drug, linear release of HST and MST was obtained. The release behavior of the NUDDMat system loaded with a drug having $\mathrm{pH}$-independent solubility was shown to be consistent in $\mathrm{pH} 1.2,4.5$ and 6.8 media. Based on these results, feasibility of the NUDDMat platform by powder layering was demonstrated using drugs having different physico-technological characteristics. Moreover, its ability to generate zero-order release was proved in the case of drugs with water solubility in a relatively wide range.
\end{abstract}

\section{Introduction}

Zero-order release kinetics has long been studied and documented in the literature for reaching constant drug absorption rate and thereby allowing to obtain constant drug plasma levels between two successive doses. Several attempts have been reported presenting different strategies, which involve mechanical restriction of swelling, application of partial coatings and/or design of modified geometries (Colombo et al., 1990, 1987; Gazzaniga et al., 1993; Grassi et al., 2004; Kim, 1995; Sangalli et al., 1994, 1993, 2003; Siepmann and Peppas, 2001; Zema et al., 2010). Such delivery systems are designed to limit the gradual increase of the diffusion path or to progressively broad the surface at the solvent penetration front within the matrix. Another approach is based on increasing drug concentration from the outside towards the inside of the dosage unit, in order to compensate for the longer diffusion path and the smaller surface available for diffusion. These are the so-called gradient matrix systems (de Haan and Lerk, 1986b, 1986a; Dekyndt et al., 2015; Hildgen and McMullen, 1995; Huang et al., 2002; James Chang and Himmelstein, 1990; Lee, 1986, 1984; Mazzoni et al., 2019; Scott and Hollenbeck, 1991; van Bommel et al., 1991). In particular, inert or hydrophilic matrix systems having non-uniform drug distribution have been proposed, depending on the type of polymer used for controlling release. Tableted systems have been prepared as multilayer or concentric units having gradient concentration of the active ingredient (Conte and Maggi, 1996; Fassihi and Ritschel, 1993; MacLeod et al., 1999; Phaechamud, 2008; Zoglio and Carstensen, 1984). Matrices with different drug concentration inside have been attained by soaking (Forni et al., 1989). In different instances, controlled drug sedimentation was exploited via centrifugation of drug particles in a molten polymer (Hildgen and McMullen, 1995). Coated systems having non-uniform drug concentration have been prepared by spraying onto inert cores dispersions of the polymer and the drug with different concentration ratios, thus creating sequential layers with non-homogeneous drug distribution (Bogentoft and Appelgren, 1984; Dekyndt et al., 2015; Li and Tu, 1991; van Bommel et al., 1989; Van Bommel et al., 1990; Wan and Lai, 1992). Monolithic systems were 3D printed including complex structures having increasing concentrations of the release modulating materials from the periphery to the center (Deng et al., 2007; Monkhouse et al., 2001). All these approaches successfully led to in vitro zero-order drug release profiles.

Recently, a novel drug delivery system with non-uniform drug distribution was developed and named NUDDMat (Non-Uniform Drug Distribution Matrix) (Cerea et al., 2018). The system consisted of overlaid layers having drug concentrations diminishing outwards according to a descending staircase function. 
Hypromellose was selected as the swellable hydrophilic polymer. The relevant amount in the best-proved configuration of the system was constant $(30 \% \mathrm{w} / \mathrm{w})$, while the percentage of an insoluble filler (DCP) increased from the innermost to the outermost layer to compensate for the decrease in drug concentration. No drug tracer was loaded in the external layer in order to possibly mitigate the burst release phase. The system was fabricated by powder layering of predetermined drug/polymer/filler mixtures onto inert starting cores. Because powder layering involves no organic solvents nor long drying operations, thus allowing typical issues connected with liquid-based layering processes to be avoided, it was particularly advantageous in shortening the processing time as compared with previously described gradient systems (Foppoli et al., 2019, 2017). NUDDMat proved able to prolong the release and extend its linearity over a relatively long period of time.

On the basis of these premises the aim of the present study was to investigate $i$ ) the possibility of broadening the design concept of the prolonged-release hydrophilic matrix system with non-uniform drug distribution (NUDDMat) to APIs having solubility in a wide range, and ii) the feasibility of powder layering technique in application of relevant powder mixtures showing different physico-technological properties.

\section{Materials}

Lansoprazole was used as a slightly soluble tracer (SST) (Adare Pharmaceuticals, Italy, water solubility <1 $\mathrm{mg} / \mathrm{mL}$, true density $1.51 \mathrm{mg} / \mathrm{mL}$ ), acetaminophen as a moderately soluble tracer (MST) (CFM, Italy water solubility $14.1 \mathrm{mg} / \mathrm{mL}$, true density $1.13 \mathrm{~g} / \mathrm{mL}$ ) and losartan potassium as a highly soluble tracer (HST)

90 (Adare Pharmaceuticals, Italy, freely soluble in water, true density $1.20 \mathrm{mg} / \mathrm{mL}$ ) (Foglio Bonda et al., 2016; Maroni et al., 2013; Tabatar et al., 1992; Venkateswara Reddy et al., 2014). High viscosity hypromellose (HPMC, Methocel ${ }^{\circledR} \mathrm{K} 15 \mathrm{M}$, Colorcon, Italy) (apparent viscosity, $2 \%$ in water at $20{ }^{\circ} \mathrm{C} 6138-9030 \mathrm{mPa} \cdot \mathrm{s}$, USP substitution type 2208 , true density $1.32 \mathrm{~g} / \mathrm{mL}$, sieved $<125 \mu \mathrm{m}$ ) was used as the hydrophilic swellable polymer (Colorcon, 2000; Qi et al., 2003; Wu et al., 2005). Dibasic calcium phosphate dihydrate (DCP, Emcompress ${ }^{\circledR}$ premium powder, JRS, Germany, true density $2.21 \mathrm{~g} / \mathrm{mL}$ ) was employed as a diluent (Roberts and Rowe, 1985). Fumed silica (Aerosil ${ }^{\circledR} 200$, Evonik, Germany) was added as a glidant to the powder mixtures, and povidone (PVP, Kollidon ${ }^{\circledR} 30$, BASF, Germany, true density $1.11 \mathrm{~g} / \mathrm{mL}$ ) was used as the binder in aqueous solution (Marsac et al., 2006). Microcrystalline cellulose (MCC) nonpareils having nominal diameter of $850 \mu \mathrm{m}$ were employed as starting cores (Cellets ${ }^{\circledR} 700$, Pharmatrans-Sanaq, Switzerland, true density 1.46 g/L) (Sun, 2005). Polysorbate 80 (TEGO SMO 80, A.C.E.F., Italy) was used for preventing aggregation of powders during particle size analysis.

\section{Methods}

\subsection{Physico-techonological characterization of drug tracers}

105 Mean particle size and size distribution of tracers were measured by single particle optical sensing (SPOS) technique using Accusizer C770 (PSS Inc. Santa Barbara, USA) equipped with an autodiluting system (Autodiluter PAT, PSS Inc. Santa Barbara, CA, USA). HST, MST, SST and DCP powders were suspended in $1 \mathrm{~mL}$ of filtered saturated aqueous solution $(0.22 \mu \mathrm{m}$ nylon filter, Millipore) containing $0.5 \%(\mathrm{w} / \mathrm{v})$ of polysorbate 80 . HPMC was tested in absolute ethanol $(\geq 99.5 \%)$. Analyses were performed in triplicate and size was expressed as the mean diameter and Span.

Span $=\frac{d_{10}-d_{90}}{d_{50}}$

Specific surface area was measured by means of SA3100 Surface Area Analyzer (Beckman Coulter, UK) according to the BET method using $\mathrm{N}_{2}$ as adsorbate gas (USP 32 Physical Test. Specific Surface Area. Volumetric Method). Prior to analysis, the samples (approximately $2 \mathrm{~g}$ ) were degassed at $90{ }^{\circ} \mathrm{C}$ under vacuum $(0.4 \mathrm{~Pa})$ for $1 \mathrm{~h}$. The measurements were carried out in triplicate. 
Compressibility index was calculated according to Eur. Pharm. 10.0 by testing approximately $50 \mathrm{~g}$ of powders using a jolting volumeter (STAV 2003, J. Engelsmann A.,D; 1250 taps) with $100 \mathrm{~mL}$ volumetric cylinder. Values reported are the average of 3 determinations. Flowability properties were classified according to the Eur. Pharm 10.0 ranking (European Pharmacopoeia, 2019).

\subsection{Manufacturing of NUDDMat and UDDMat}

Powder blends to be layered or tableted were obtained by mixing in Turbula ${ }^{\circledR}$ (Willy A. Bachofen, Switzerland) at $24 \mathrm{rpm}$ for $20 \mathrm{~min}$.

Powder layering was performed by a fluid bed apparatus (GPGC 1.1, Glatt, Germany) equipped with rotor insert. The operating conditions set up were as follows: nozzle port size $1.2 \mathrm{~mm}$, inlet air temperature $30^{\circ} \mathrm{C}$, product temperature $26-28{ }^{\circ} \mathrm{C}$, outlet air temperature $23{ }^{\circ} \mathrm{C}$, air flow $70 \mathrm{~m}^{3} / \mathrm{h}$, nebulization air pressure $2 \mathrm{bar}$, product pressure $1100 \mathrm{~Pa}$, disk rotation speed $700 \mathrm{rpm}$, liquid binder feeding rate $12 \mathrm{~g} / \mathrm{min}$, powder feeding rate $20 \mathrm{~g} / \mathrm{min}$. For any layering step, $500 \mathrm{~g}$ of starting substrate, either inert seeds or intermediate layered units ( 1 to 4 layers), was loaded into the processing chamber. Powder addition was carried out via a forced powder feeder while spraying in a continuous mode a binding solution onto preheated substrate. The amount of powder formulations to be layered was established so as to reach the target $315 \mu \mathrm{m}$ increase in thickness by assuming a 5\% layer porosity and a $90 \%$ process yield. The diameter and weight of the coated units were regularly checked in-process by withdrawing samples $(n=100)$ at prefixed time intervals. After each layering step, a drying phase was performed at the inlet air temperature of $60^{\circ} \mathrm{C}$ for $30 \mathrm{~min}$, and process yield was calculated as the percentage layered units/starting materials (seeds, layering powders, binder) weight ratio.

The UDDMat (Uniform Drug Distribution Matrix) system containing MST was manufactured by tableting, using a rotary tablet press (mod. AM8S, Officine Meccaniche Ronchi, Italy) equipped with $4 \mathrm{~mm}$ diameter concave punches ( $4 \mathrm{~mm}$ curvature radius) at a $7 \mathrm{kN}$ compression force (batch size $50 \mathrm{~g}$ ). The nominal weight of the matrices was $45 \mathrm{mg}$. The composition of the powder blend employed was the same as in 5-layer NUDDMat MST, as reported in Table 1.

\subsection{Physico-technological characterization of layered and tableted matrices}

Layered units $(n=100)$ and tablets $(n=20)$ were checked for weight and for thickness and/or diameter by a precision caliper (CD 150, Mitutoyo, Italy). The aspect ratio was calculated dividing the maximum by the minimum diameter of each unit, as measured by digital microscope (Dino Lite Pro AM 413T, Italeco, Italy) $(n=20)$. Friability was assessed according to Eur. Pharm. 10.0 by a friabilometer (mod. EF-2, Electrolab, Italy) rotating at $25 \mathrm{rpm}$ for $4 \mathrm{~min} .6 .5 \mathrm{~g}$ of units was used for the measurements. The data obtained were expressed as loss-on-weight percentages. Crushing strength $(\mathrm{n}=10)$ was measured by the equipment reported in Eur. Pharm. 10.0 (mod. T3, Erweka, Germany). Porosity $(E)$ of units, expressed as percentage, was

150 calculated according to the equation:

$E(\%)=\left[1-\frac{V_{t}}{V_{b}}\right] \cdot 100$

where $\mathrm{V}_{\mathrm{t}}$ is the average true volume and $\mathrm{V}_{\mathrm{b}}$ is the mean bulk volume.

155 Cross-section morphology was analyzed by a scanning electron microscope (SEM; FEI, Carl Zeiss, Germany). Samples were gold-sputtered using a plasma evaporator under vacuum, and photomicrographs were acquired at an accelerated voltage of $10 \mathrm{kV}$ at differing magnifications. 


\subsection{Release testing and data analysis of layered and tableted matrices}

160 Weighed samples approximately corresponding to an overall amount of $50 \mathrm{mg}$ of tracer were tested for release by a Eur. Pharm. 10.0 dissolution apparatus (mod. 2100B, Distek Italia, Italy) equipped with rotating baskets using $900 \mathrm{~mL}$ of fluid thermostated at $37 \pm 0.5^{\circ} \mathrm{C}$ and stirred at $100 \mathrm{rpm}$. Purified water, hydrochloric acid solution $\mathrm{pH} 1.2$ and phosphate buffer $\mathrm{pH} 4.5$ or 6.8 were employed as the media. Release tests were performed in triplicate. Tracers were assayed by spectrophotometer (Lambda 25, Perkin Elmer Italia, Italy) at the wavelength of 250, 243 and $284 \mathrm{~nm}$ for losartan potassium, acetaminophen and lansoprazole, respectively, after verifying agreement with results acquired by HPLC validated methods (Cerea et al., 2018; Chambers et al., 2005; Del Curto et al., 2014; United States Pharmacopeia Convention, 2017).

For statistical comparison of release profiles, similarity factor $f_{2}$ was applied. $f_{2} \geq 50$ (50-100) indicated similarity (Administration Centre For Drug US Department of Health and Human Services Food and Drug and CDER Evaluation And Research, 1997).

UDDMat release data were analyzed according to the equation:

$\frac{M_{t}}{M_{\infty}}=k t^{n}$

175 where $M_{t} / M_{\infty}$ is the drug fraction released at time $t, n$ exponent indicates the mechanism/kinetics of release and $k$ is a constant incorporating structural and geometrical characteristics of the matrix. Analysis of data was performed in the $\mathrm{M}_{\uparrow} / \mathrm{M}_{\infty}<0.60$ curve portion, calculating confidence intervals (c.i.) at $95 \%$ (Colombo et al., 1985; Korsmeyer et al., 1983; Sinclair and Peppas, 1984).

NUDDMat release data were also analyzed by Eq. (3), in the $\mathrm{M}_{\mathrm{t}} / \mathrm{M}_{\infty}<0.90$ curve portion. In this case, $n$ values were used in a merely descriptive way to highlight progressive shift of kinetics toward linearity. $n=1$ indicated apparent zero-order release. Values $<0.5$ and $>1$ could also be taken into account.

The extent of linearity of release profiles was evaluated through the Durbin-Watson statistics, which enables to identify zero-order portions in each curve (Durbin and Watson, 1950; van der Voet et al., 1983) .

\section{Results and discussion}

For the purpose of evaluating the impact of APIs with different solubility on powder layering feasibility and the release performance of the resulting units, the matrix design was maintained as previously set up (Cerea et al., 2018), including:

i) 5 layers having nominal thickness of $315 \mu \mathrm{m}$ (starting core $850 \mu \mathrm{m}$ nominal diameter);

ii) decreasing concentration of the drug tracer from the innermost $\left(1^{\text {st }}\right)$ layer toward the outermost $\left(5^{\text {th }}\right)$ one;

iii) constant concentration of hydrophilic swellable release-controlling polymer throughout all layers $(30 \% \mathrm{w} / \mathrm{w})$;

iv) increasing concentration of an insoluble filler (DCP) from the $1^{\text {st }}$ to the $5^{\text {th }}$ layer;

v) no drug tracer in the $5^{\text {th }}$ layer in order to counteract the burst release.

The concentration of drug tracer was decreased between contiguous layers from the inside toward the outside of the matrix reflecting a descending staircase function, thus tending to an apparent linear mode, from $65 \%$ in the $1^{\text {st }}$ layer to $0 \%$ in the $5^{\text {th }}$ one. Fig. 1 reports a schematic outline of the NUDDMat system, while Fig. 2 presents the concentration profiles for the three main components, i.e. the tracer, polymer and filler. 


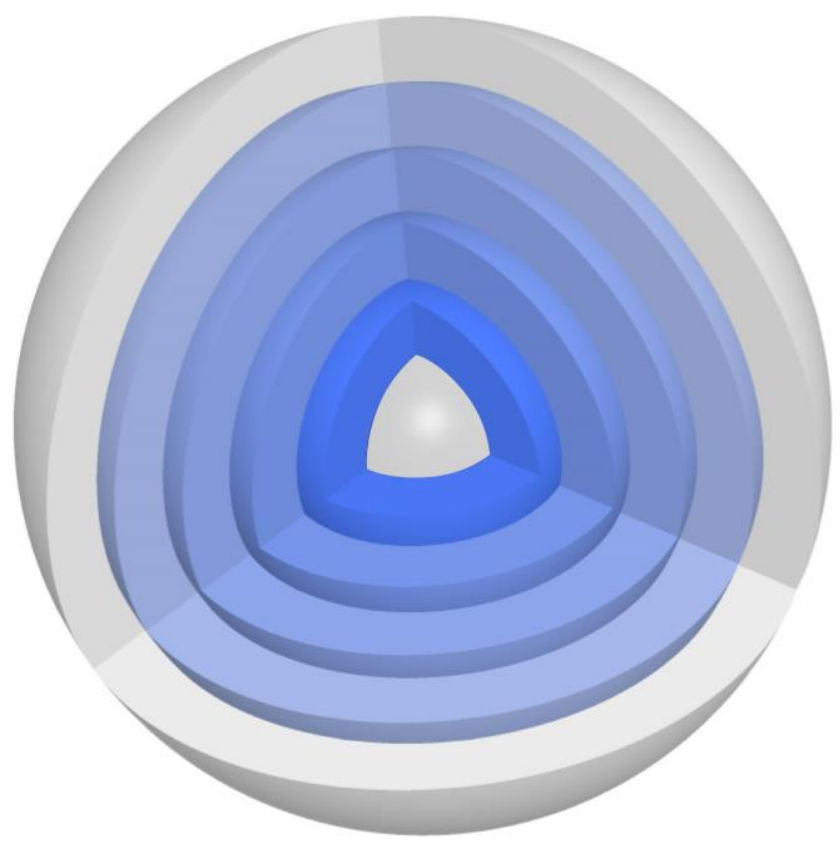

Fig. 1. Schematic 3D representation of the NUDDMat system. Drug tracer concentration is indicated by color intensity.

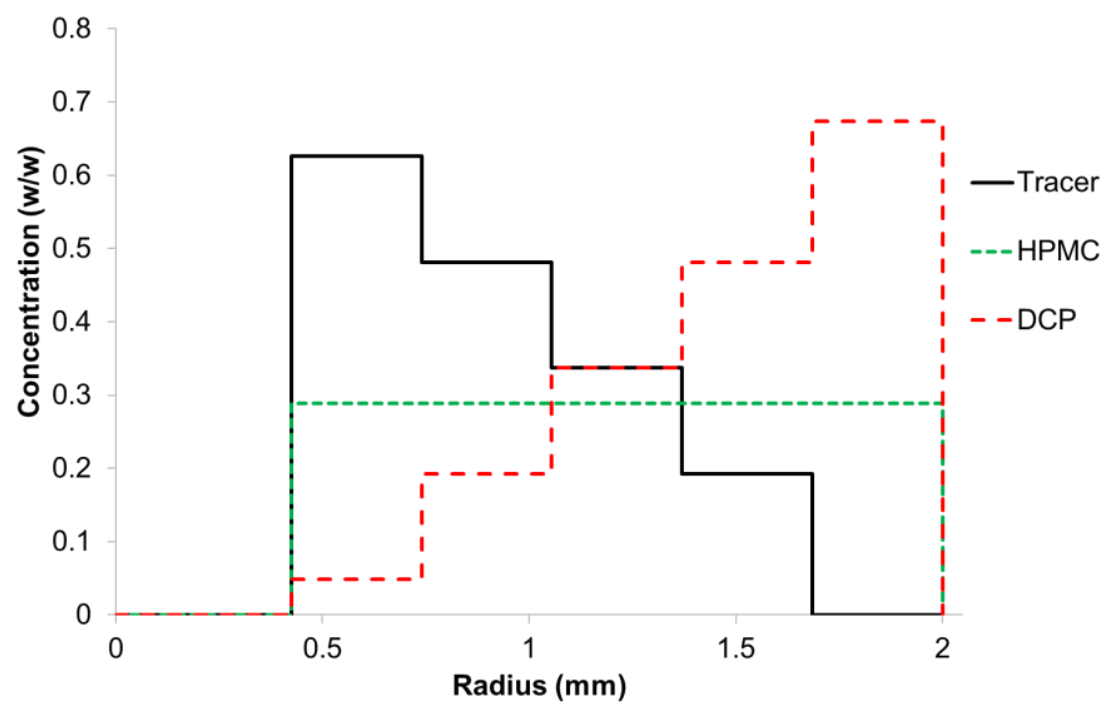

Fig. 2. Theoretical concentration patterns for drug tracer, polymer and filler along the radius of the NUDDMat system. 
215 Relying on the above-presented design concept, the percentage compositions of the powder blends reported in Table 1 were used.

\section{Table 1}

Nominal composition of the different powder blends

\begin{tabular}{|c|c|c|c|c|}
\hline & Layer & Tracer $(\%)$ & HPMC (\%) & $\mathrm{DCP}(\%)$ \\
\hline \multirow{5}{*}{$\begin{array}{l}\text { NUDDMat }{ }^{\#} \\
\text { (Non-uniform Drug Distribution } \\
\text { Matrices) }\end{array}$} & $1^{\text {st }}$ & 65 & 30 & 5 \\
\hline & $2^{\text {nd }}$ & 50 & 30 & 20 \\
\hline & $3^{\text {rd }}$ & 35 & 30 & 35 \\
\hline & $4^{\text {th }}$ & 20 & 30 & 50 \\
\hline & $5^{\text {th }}$ & - & 30 & 70 \\
\hline $\begin{array}{l}\text { UDDMat }{ }^{\# \#} \\
\text { (Uniform Drug Distribution } \\
\text { Matrices) }\end{array}$ & - & 17 & 30 & 53 \\
\hline
\end{tabular}

Three small molecules having different aqueous solubility were selected: losartan potassium (HST), acetaminophen (MST) and lansoprazole (SST), used as highly soluble, moderately soluble and slightly soluble drug tracers, respectively.

Because powder layering involves the use of relatively small-sized powders, generally not above $150 \mu \mathrm{m}$, in order to improve the packing of particles in layer formation, fine grade active pharmaceutical ingredients (APIs) and excipients were employed (Table 2). The particle dimensions of HST were relatively larger as compared with MST and SST, the latter exhibiting a relatively narrow range and the highest specific surface area. The relatively high surface area of HPMC in spite of the large particle size might be due to the great porosity with respect to other materials. The morphological particle characteristics of the APIs selected were well evident by SEM analysis, which pointed out agglomeration of SST powder (Fig. 3). The APIs were categorized according to the Eur. Pharm. 10.0 classification as "very very poor" flowable powders

235 (European Pharmacopoeia, 2019). DCP and HPMC, also employed as fine powders, proved to only slightly enhance the flow properties of the resulting mixtures. 


\section{Table 2}

240 Physico-technological characteristics of drug tracers, polymer and filler

\begin{tabular}{cccc}
\hline Tracer & Particle size & Specific surface area & Compressibility index \\
\hline HST & $103.59 \mu \mathrm{m}(42.07$ Span $)$ & $0.326 \mathrm{~m}^{2} / \mathrm{g}(0.012 \mathrm{sd})$ & $38.0 \%(4.8 \mathrm{sd})$ \\
MST & $47.02 \mu \mathrm{m}(13.53 \mathrm{Span})$ & $0.560 \mathrm{~m}^{2} / \mathrm{g}(0.016 \mathrm{sd})$ & $42.5 \%(5.3 \mathrm{sd})$ \\
SST & $12.27 \mu \mathrm{m}(1.16 \mathrm{Span})$ & $2.105 \mathrm{~m}^{2} / \mathrm{g}(0.025 \mathrm{sd})$ & $50.1 \%(7.1 \mathrm{sd})$ \\
HMPC & $75.55 \mu \mathrm{m}(4.48$ Span $)$ & $1.520 \mathrm{~m}^{2} / \mathrm{g}(0.035 \mathrm{sd})$ & $31.1 \%(2.4 \mathrm{sd})$ \\
DPC & $13.2 \mu \mathrm{m}(3.25 \mathrm{Span})$ & $1.250 \mathrm{~m}^{2} / \mathrm{g}(0.045 \mathrm{sd})$ & $25.2 \%(2.7 \mathrm{sd})$ \\
\hline
\end{tabular}

a)

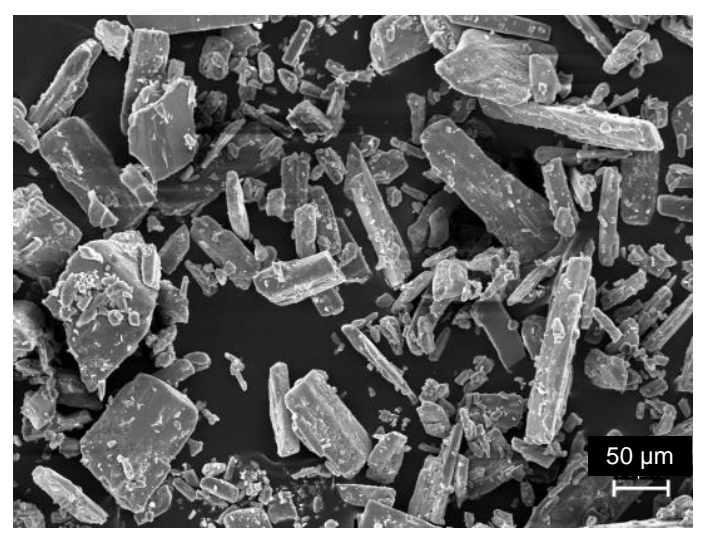

250

b)

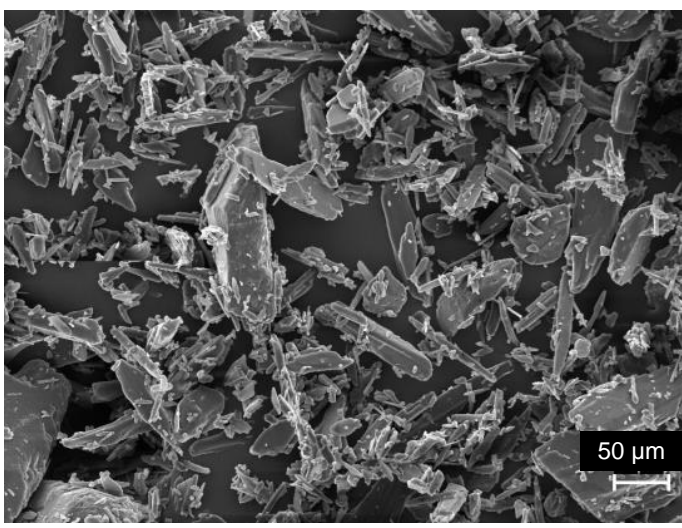

c)

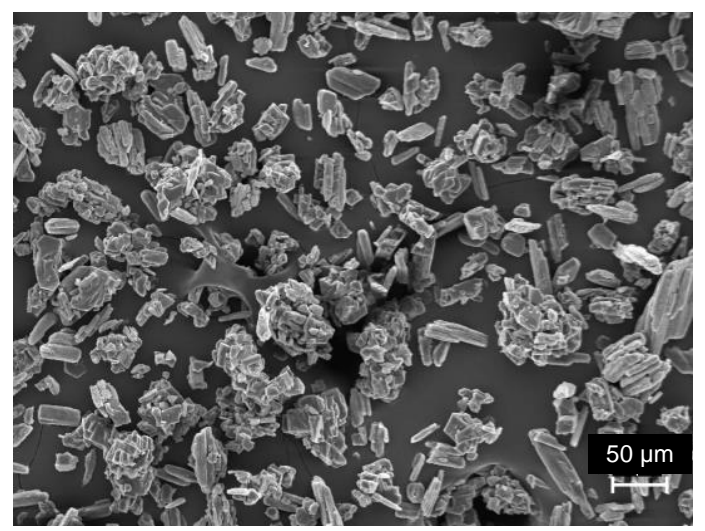

Fig. 3. SEM photomicrographs of HST (a), MST (b) and SST (c) powders. 
As a first step, it was necessary to define the nominal percentage composition of the NUDDMat systems. In this respect, preset tracer:HPMC weight ratios, as reported in Table 1, were relied on to calculate the formula of the layers, also accounting for the amounts of fumed silica (0.72\%) and of PVP (3.00\%). The percentage of PVP was derived from the fixed 5:3 weight ratio between layered powder and 5\% w/w binding solution. The total mass of materials needed for each layer was calculated on the basis of the average true density of the formulation by assuming a layer porosity of $5 \%$ (Table 3 ). The values reported were subsequently used to establish the amounts of powder and binding solution to be loaded to reach the desired thickness for each layer.

\section{Table 3}

Nominal diameter, volume, weight, drug load and drug concentration, for each layer and cumulative, in NUDDMat systems containing different drug tracers.

\begin{tabular}{|c|c|c|c|c|c|c|c|c|}
\hline & & $\begin{array}{l}\text { core } \\
\text { seed }\end{array}$ & 1-layer & 2-layer & 3-layer & 4-layer & 5-layer/2 & 5-layer \\
\hline Cumulative diameter (mm) & $\mathrm{HST} / \mathrm{MST} / \mathrm{SST}$ & 0.85 & 1.48 & 2.11 & 2.74 & 3.37 & 3.69 & 4.00 \\
\hline Layer volume $\left(\mathrm{mm}^{3}\right)$ & HST/MST/SST & - & 1.38 & 3.22 & 5.85 & 9.26 & 6.16 & 13.46 \\
\hline Cumulative volume $\left(\mathrm{mm}^{3}\right)$ & HST/MST/SST & 0.32 & 1.70 & 4.92 & 10.77 & 20.03 & 26.19 & 33.49 \\
\hline \multirow{3}{*}{$\begin{array}{l}\text { Layer weight } \\
\qquad(\mathrm{mg})\end{array}$} & HST & - & 1.64 & 4.28 & 8.58 & 14.87 & 11.02 & 24.10 \\
\hline & MST & - & 1.58 & 4.17 & 8.45 & 14.75 & 11.02 & 24.10 \\
\hline & SST & - & 1.89 & 4.73 & 9.16 & 15.40 & 11.02 & 24.10 \\
\hline \multirow{3}{*}{$\begin{array}{l}\text { Cumulative weight } \\
\qquad(\mathrm{mg})\end{array}$} & HST & 0.50 & 2.13 & 6.41 & 14.99 & 29.86 & 40.88 & 53.96 \\
\hline & MST & 0.50 & 2.08 & 6.25 & 14.70 & 29.45 & 40.47 & 53.55 \\
\hline & SST & 0.50 & 2.39 & 7.12 & 16.28 & 31.67 & 42.69 & 55.77 \\
\hline \multirow{3}{*}{$\begin{array}{l}\text { Layer drug load } \\
\qquad(\mathrm{mg})\end{array}$} & HST & - & 1.02 & 2.06 & 2.89 & 2.86 & 0.00 & 0.00 \\
\hline & MST & - & 0.99 & 2.01 & 2.85 & 2.84 & 0.00 & 0.00 \\
\hline & SST & - & 1.18 & 2.28 & 3.09 & 2.96 & 0.00 & 0.00 \\
\hline \multirow{3}{*}{$\begin{array}{l}\text { Cumulative drug load } \\
\qquad(\mathrm{mg})\end{array}$} & HST & - & 1.02 & 3.08 & 5.97 & 8.84 & 8.84 & 8.84 \\
\hline & MST & - & 0.99 & 3.00 & 5.84 & 8.68 & 8.68 & 8.68 \\
\hline & SST & - & 1.18 & 3.46 & 6.55 & 9.51 & 9.51 & 9.51 \\
\hline \multirow{3}{*}{$\begin{array}{l}\text { Layer drug concentration } \\
\qquad\left(\mathrm{mg} / \mathrm{mm}^{3}\right)\end{array}$} & HST & - & 0.60 & 0.42 & 0.27 & 0.14 & 0.00 & 0.00 \\
\hline & MST & - & 0.72 & 0.62 & 0.49 & 0.31 & 0.00 & 0.00 \\
\hline & SST & - & 0.86 & 0.71 & 0.53 & 0.32 & 0.00 & 0.00 \\
\hline
\end{tabular}


The $315 \mu$ m-thick layers included in the system design involved 5 sequential processing steps. For each one, the starting batch size was of $500 \mathrm{~g}$, of either inert seeds or intermediate layered units. The quantity of powder required for the single layers was calculated based on the values reported in Table 3 and the assumption of a $90 \%$ process yield, previously assessed by averaging the results from preliminary trials. The binding solution having the established PVP concentration was proved to enable proper adhesion of particles onto the substrate regardless of remarkable differences in the solubility of the drug tracers selected. The operating parameters set turned out to be suited for all powder formulations to be layered. Particularly, the 5:3 weight ratio between powder feeding rate $(20 \mathrm{~g} / \mathrm{min})$ and liquid binder spraying rate $(12 \mathrm{~g} / \mathrm{min})$ was maintained during the whole process with the 3 tracers investigated.

A drying phase was carried out when the loading of the whole amount of powder was accomplished. The resulting units, both intermediate and final, exhibited size and weight uniformity, satisfactory aspect ratio, relatively low porosity and negligible friability (Table 4a). Pictures of the uncoated seeds and of systems with increasing layers containing the different tracers are reported in Fig. 4, and SEM photomicrographs are 295 shown in Fig. 5.

The increases in weight and diameter were close to the expected values, although slightly higher data were obtained because the process yield was in all cases found greater than the assumed $90 \%$.

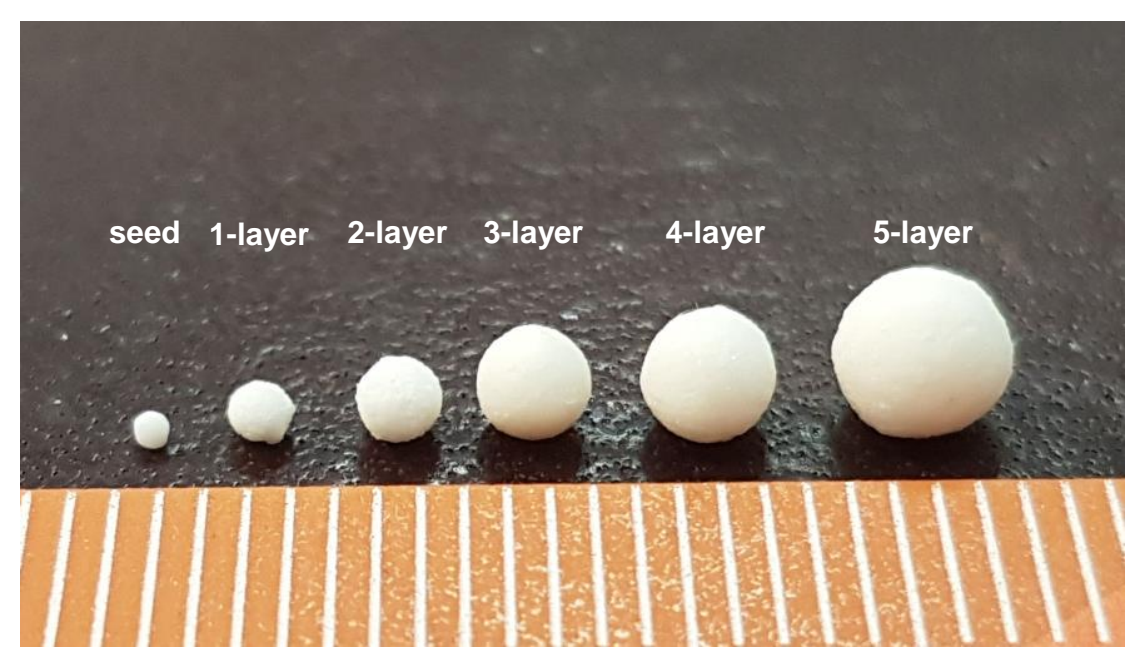

Fig. 4. Units ranging from core seed to final NUDDMat system containing HST (scale in millimeters). 
a)
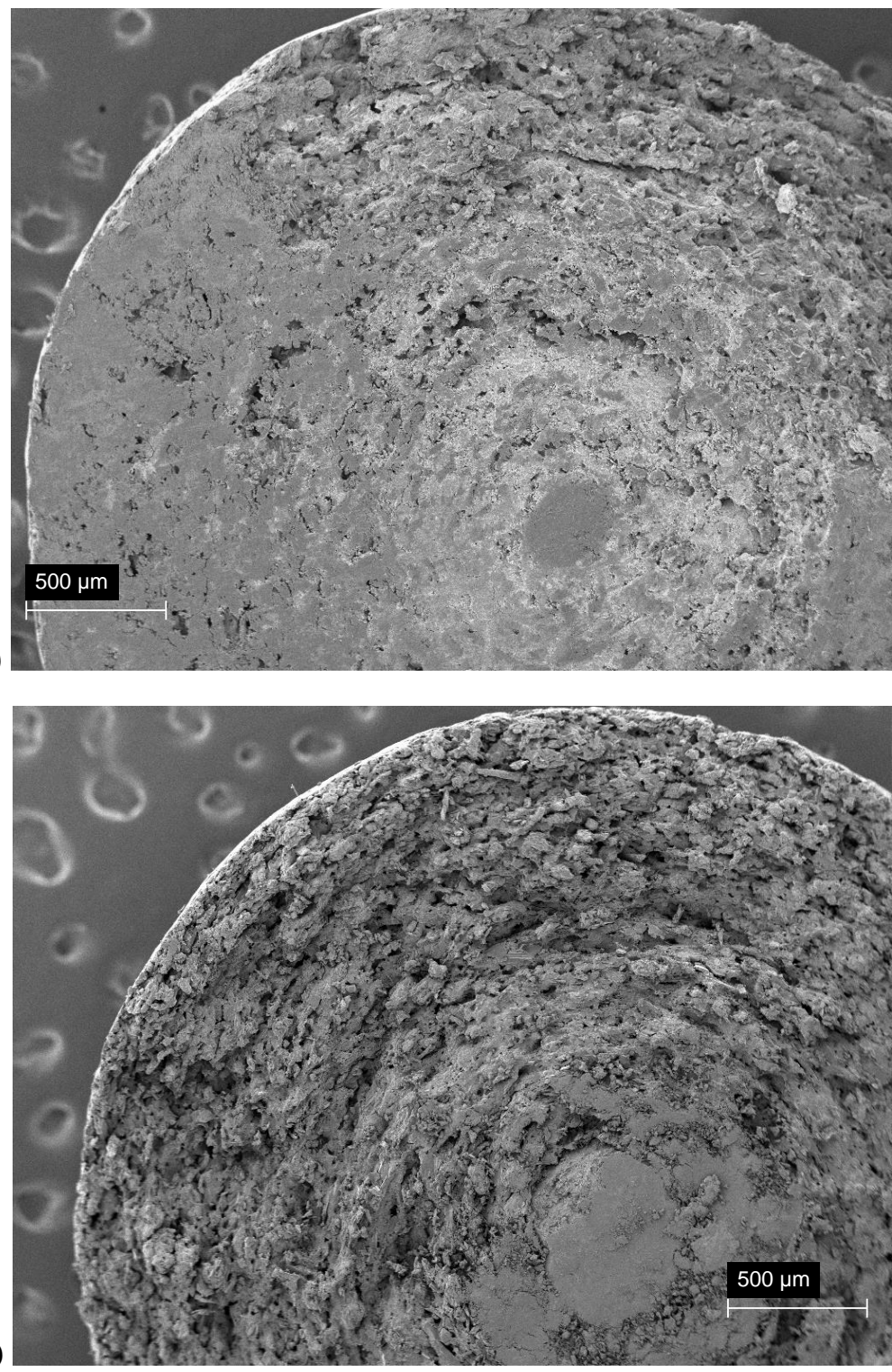

b)

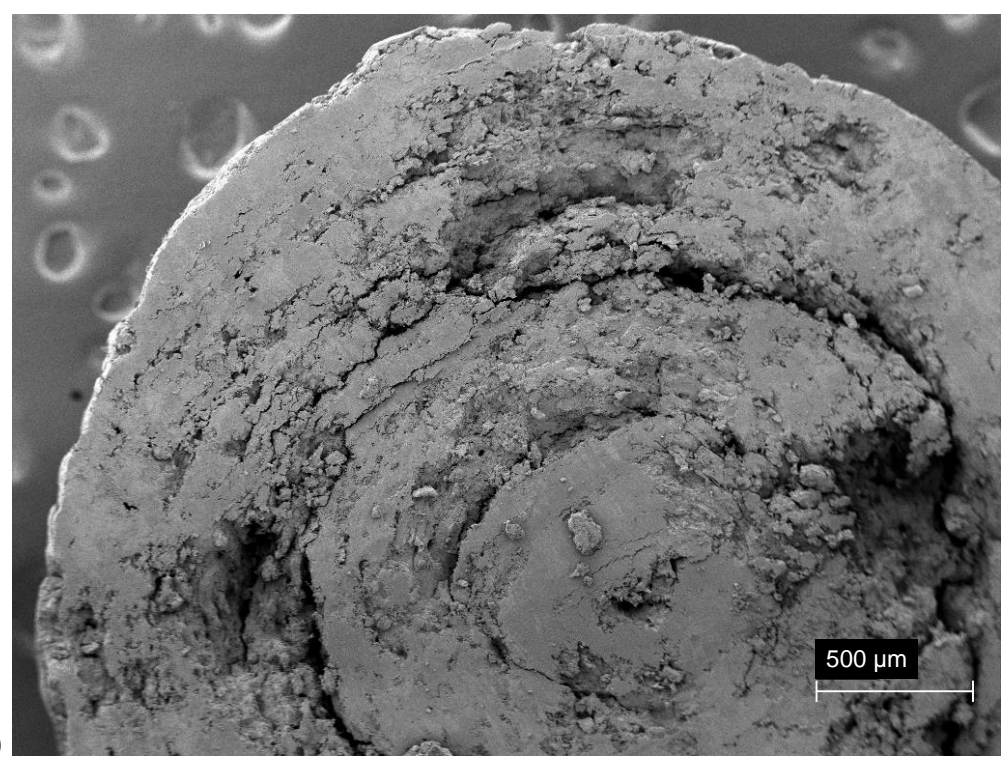

Fig. 5. SEM photomicrographs of cross-sectioned NUDDMat systems containing HST (a), MST (b) e SST (c). 


\section{Table 4}

Physico-technological characteristics of NUDDMat systems.

a)

\begin{tabular}{ccccccc}
\hline $\begin{array}{c}\text { NUDDMat } \\
\text { unit }\end{array}$ & $\begin{array}{c}\text { Weight } \\
(m g \pm \text { s.d. })\end{array}$ & $\begin{array}{c}\text { Diameter } \\
(m m \pm \text { s.d. })\end{array}$ & $\begin{array}{c}\text { Porosity } \\
(\%)\end{array}$ & $\begin{array}{c}\text { Aspect ratio } \\
(\text { value } \pm \text { s.d. })\end{array}$ & $\begin{array}{c}\text { Crushing } \\
\text { strength } \\
(N \pm \text { s.d. })\end{array}$ & $\begin{array}{c}\text { Friability } \\
(\%)\end{array}$ \\
\hline HST & $55.01 \pm 1.28$ & $4.09 \pm 0.08$ & 6.25 & $1.11 \pm 0.03$ & $125 \pm 12$ & 0.11 \\
MST & $54.05 \pm 1.79$ & $4.11 \pm 0.08$ & 7.35 & $1.09 \pm 0.02$ & $146 \pm 8$ & 0.13 \\
SST & $56.05 \pm 1.59$ & $4.03 \pm 0.08$ & 8.21 & $1.15 \pm 0.04$ & $101 \pm 6$ & 0.21 \\
\hline
\end{tabular}

b)

\begin{tabular}{|c|c|c|c|c|c|c|}
\hline $\begin{array}{l}\text { NUDDMat } \\
\text { unit }\end{array}$ & $\begin{array}{c}\text { Weight } \\
(m g \pm \text { s.d.) }\end{array}$ & $\begin{array}{l}\text { Diameter } \\
(m m \pm \text { s.d. })\end{array}$ & $\begin{array}{c}\text { Porosity } \\
\text { (\%) }\end{array}$ & $\begin{array}{l}\text { Aspect ratio } \\
\text { (value } \pm \text { s.d.) }\end{array}$ & $\begin{array}{l}\text { Crushing } \\
\text { strength } \\
(N \pm \text { s.d. })\end{array}$ & $\begin{array}{c}\text { Friability } \\
\text { (\%) }\end{array}$ \\
\hline HST/2 & $42.02 \pm 1.08$ & $3.71 \pm 0.04$ & 5.89 & $1.10 \pm 0.03$ & $101 \pm 10$ & 0.15 \\
\hline $\mathrm{MST} / 2$ & $41.45 \pm 1.25$ & $3.68 \pm 0.06$ & 7.51 & $1.02 \pm 0.03$ & $110 \pm 10$ & 0.13 \\
\hline $\mathrm{SST} / 2$ & $42.58 \pm 1.56$ & $3.71 \pm 0.04$ & 8.50 & $1.01 \pm 0.02$ & $90 \pm 6$ & 0.18 \\
\hline
\end{tabular}

In the SEM images of cross-sectioned units, the internal structure appeared different depending on the concerned API. In no case, an onion-like aspect, which might have resulted from successive application of the diverse formulations, could clearly be seen.

The release profiles of units provided with 1 to 5 layers are reported in Fig. 6. With increasing number of layers applied, the release was progressively slowed down. A lower release rate was observed according to the solubility of the drug tracer.

HST- (NUDDMat HST) and MST-containing (NUDDMat MST) systems displayed analogous release profiles both for intermediate units having 1 to 4 layers and for final systems. In the units provided with 1 layer only, with all tracers considered, an effective matrix structure could not be formed due to the very limited amount of polymer, and an early breakup occurred.

SST-containing units with 1 to 4 layers showed sigmoidal release profiles. This may be due to the relatively low dissolution rate of such a drug tracer, which would ultimately be preceded by formation of a swollen HPMC barrier effective in hindering release.

The application of the outer layer, devoid of tracer, always led to an initial slower release phase, which was especially evident in the case of the system containing SST (NUDDMat SST), with a duration of 4-5 h. In the latter case, because of the poor API solubility, erosion may have indeed prevailed over diffusion as the release-controlling mechanism (Lee, 1986). This seemed to be supported by the increase in the variability of release data that was observed for NUDDMat SST systems with 5 layers. The system containing SST yielded an apparent zero-order release in the absence of the $5^{\text {th }}$ layer (NUDDMat SST 4-layer). In this 
respect, modulation of the concentration gradient and/or modification of the layer configuration as a function of drug solubility could represent a viable strategy to pursue zero-order release.

335 Data were analyzed by exponential Eq. (3), here applied for descriptive purposes only, confirming the above considerations. In particular, an increase in $n$ values for NUDDMat HST and NUDDMat MST indicated progressive shifting of the profiles towards zero-order kinetics, while $n$ largely $>1$ relevant to NUDDMat SST was associated with deferred release (Table 5). With NUDDMat HST and NUDDMat MST systems (5layer units), $n$ slightly $>1$ was found, pointing out initially lowered rate of release and consequent tendency 340 to sigmoidal curves. In order to address such an issue, the thickness of the outer layer with no tracer was halved to give NUDDMat HST/2, NUDDMat MST/2 and NUDDMat SST/2 (Table 4b). Porosity, aspect ratio and mechanical resistance were not altered with respect to the original NUDDMat systems, while the release performance was changed as shown in Fig. 7.

As pursued, through reduction of the thickness of the $5^{\text {th }}$ layer to $157.5 \mu \mathrm{m}$, it was possible to attain linear 345 release of HST and MST. Indeed, the resulting $n$ values were close to unity. Moreover, through the DurbinWatson statistics, linearly released fractions in the range 0.20 to 0.88 , between 1 and $5 \mathrm{~h}$, were calculated from NUDDMat HST/2, while with NUDDMat MST/2 release was found linear from 0.17 to 0.87 fractions, between 1 and $6 \mathrm{~h}$. On the other hand, in the case of NUDDMat SST/2 this strategy proved only useful to shorten the starting lag phase, and the release pattern still appeared sigmoidal as testified by the corresponding $n$ value. 
a)

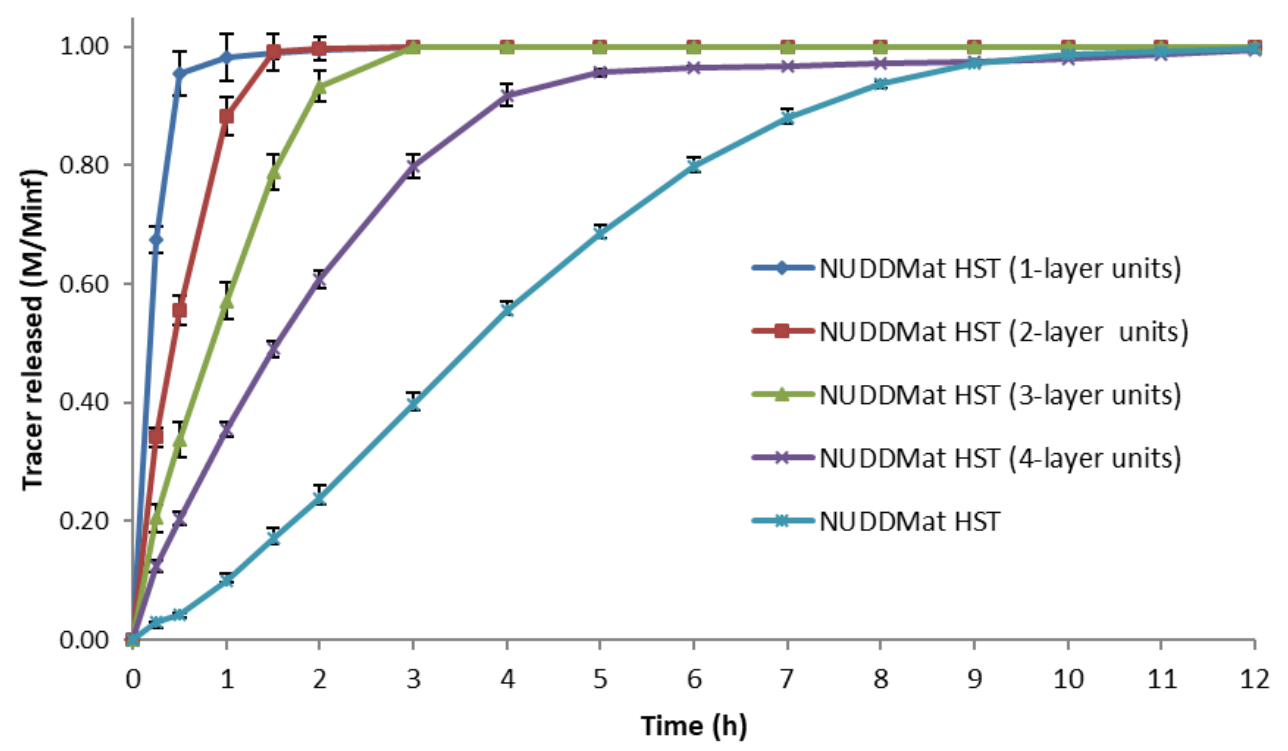

b)

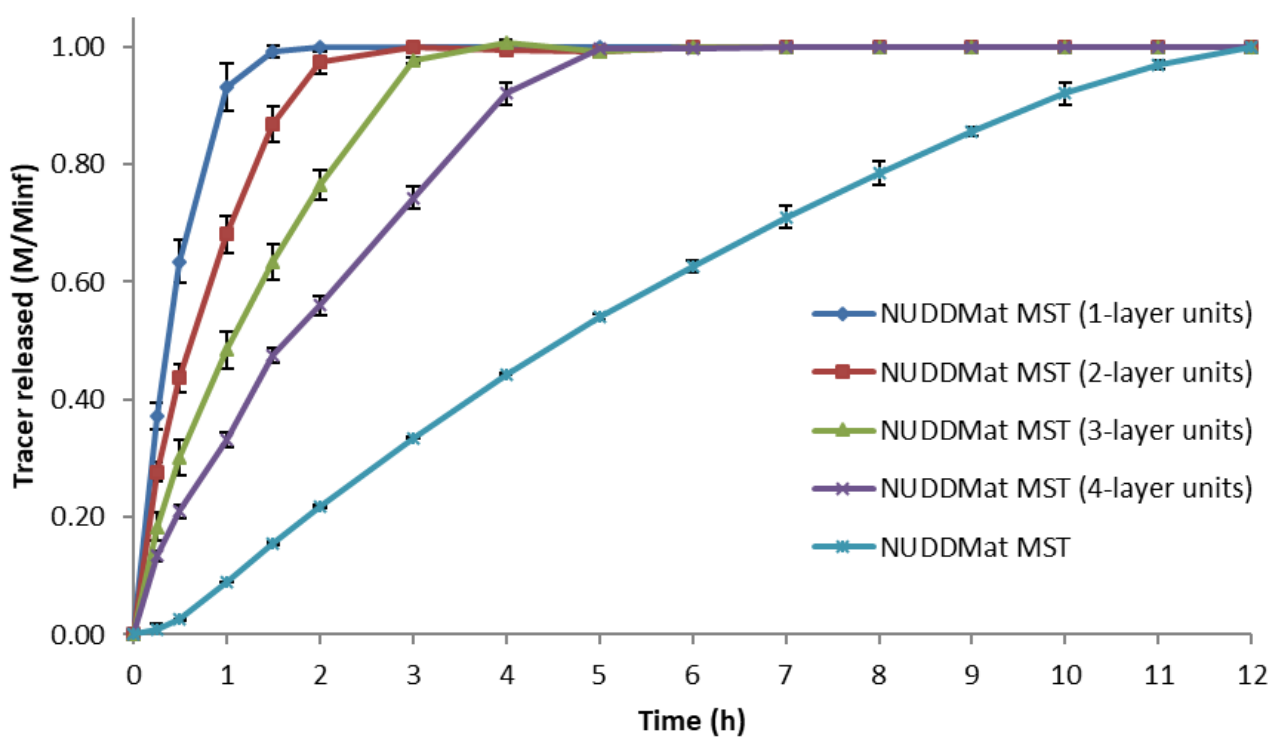

c)

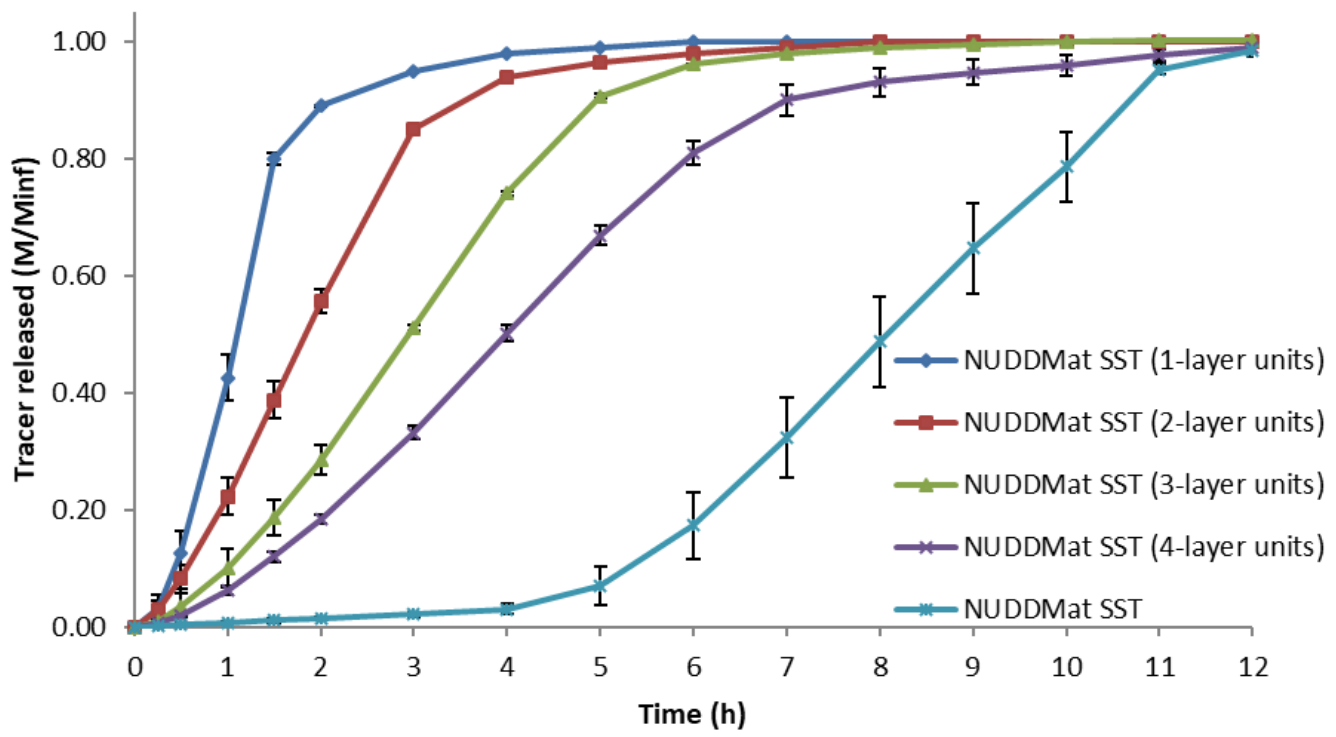

Fig. 6. Release profiles from NUDDMat systems containing HST (a), MST (b) and SST (c). 


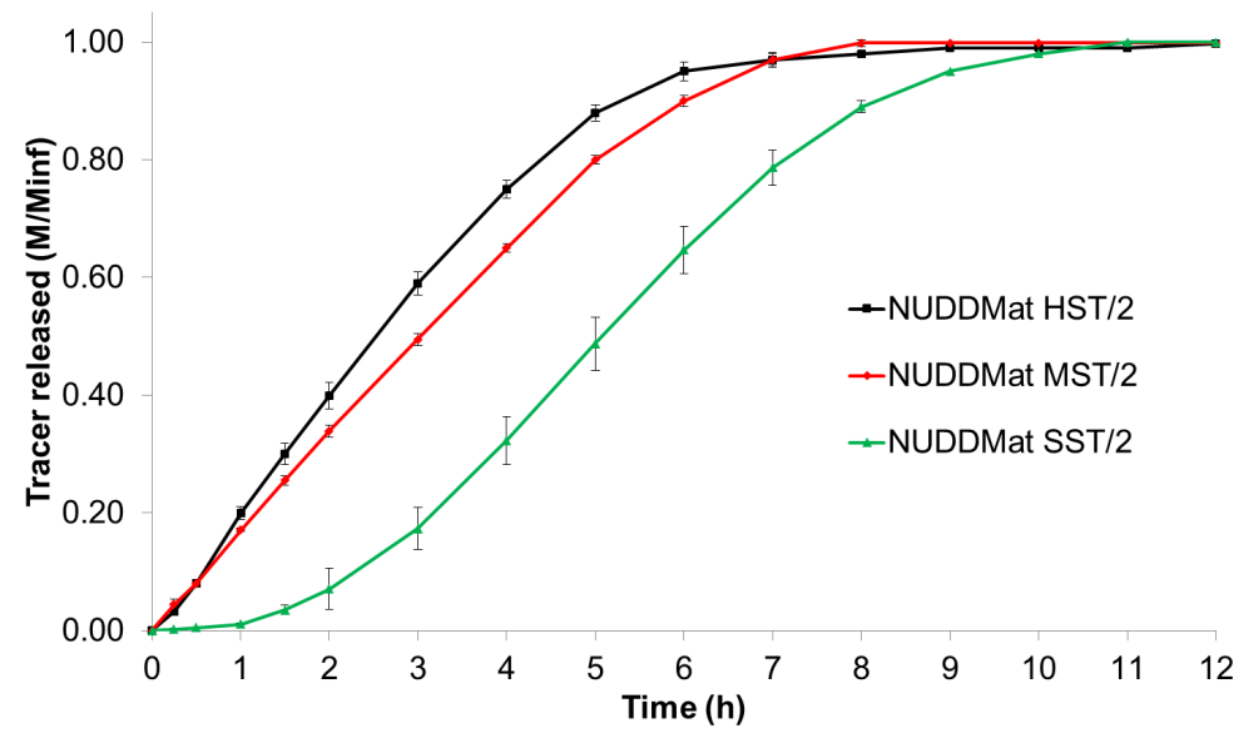

Fig. 7. Release profiles from NUDDMat systems with $5^{\text {th }}$ layer having $157.5 \mu \mathrm{m}$ thickness. 
Table 5.

Release parameters according to exponential Eq. (3).

\begin{tabular}{lllll}
\hline & $\mathrm{n}$ & confidence limit 95\% & $\mathrm{k}$ & $\mathrm{R}^{2}$ \\
\hline NUDDMat HST 1-layer & $*$ & $*$ & $*$ & $*$ \\
NUDDMat HST 2-layer & 0.685 & 0.001 & 0.886 & 1.000 \\
NUDDMat HST 3-layer & 0.750 & 0.001 & 0.575 & 1.000 \\
NUDDMat HST 4-layer & 0.761 & 0.001 & 0.354 & 1.000 \\
NUDDMat HST/2 & 1.069 & 0.035 & 0.170 & 0.993 \\
NUDDMat HST & 1.086 & 0.001 & 0.060 & 0.992 \\
\hline NUDDMat MST 1-layer & $*$ & $*$ & $*$ & $*$ \\
NUDDMat MST 2-layer & 0.640 & 0.002 & 0.676 & 1.000 \\
NUDDMat MST 3-layer & 0.686 & 0.003 & 0.480 & 1.000 \\
NUDDMat MST 4-layer & 0.697 & 0.001 & 0.345 & 0.999 \\
NUDDMat MST/2 & 0.982 & 0.002 & 0.168 & 1.000 \\
NUDDMat MST & 1.154 & 0.002 & 0.081 & 0.999 \\
\hline NUDDMat SST 1-layer & $*$ & $*$ & $*$ & $*$ \\
NUDDMat SST 2-layer & 1.374 & 0.002 & 0.209 & 0.999 \\
NUDDMat SST 3-layer & 1.531 & 0.001 & 0.096 & 0.999 \\
NUDDMat SST 4-layer & 1.510 & 0.002 & 0.060 & 0.999 \\
NUDDMat SST/2 & 1.874 & 0.003 & 0.020 & 0.993 \\
NUDDMat SST & 1.637 & 0.003 & 0.009 & 0.945 \\
\hline NUDDMat MST pH 1.2 & 1.042 & 0.074 & 0.100 & 0.997 \\
NUDDMat MST pH 4.5 & 1.197 & 0.146 & 0.077 & 0.997 \\
NUDDMat MST pH 6.8 & 1.092 & 0.146 & 0.075 & 0.945 \\
\hline UDDMat MST pH 1.2 & 0.768 & 0.037 & 0.233 & 0.999 \\
UDDMat MST pH 4.5 & 0.661 & 0.110 & 0.264 & 0.999 \\
UDDMat MST pH 6.8 & 0.615 & 0.182 & 0.998 \\
\hline
\end{tabular}

* not applicable due to rapid erosion/disintegration

A well-known advantageous feature of HPMC-based matrices, provided that the API has $\mathrm{pH}$-independent solubility, is consistent performance regardless of diverse $\mathrm{pH}$ of the environment as encountered in the gastrointestinal tract. In order to verify whether such a behavior would also apply to the proposed gradient matrix system, release was evaluated in compendial fluids having different $\mathrm{pH}$ values. Particularly, this study was conducted using NUDDMat MST, the dissolution properties of acetaminophen being unaffected by $\mathrm{pH}$. Tableted matrices with the same quali-quantitative composition of final NUDDMat MST (tracer, polymer, 400 diluent, binder), yet with uniform drug distribution (UDDMat MST), were also prepared for comparison. With both the uniform and non-uniform drug distribution systems, no influence of the fluid $\mathrm{pH}$ was observed on the release performance, as indicated by similarity factors $f_{2}$ always $>80$ within the two data sets (Fig. 8). Moreover, a clear tendency toward zero-order of NUDDMat MST release profiles was highlighted in all fluids. Notably, in the case of UDDMat systems, $n$ values hold mechanistic meaning in exponential Eq. (3), thus pointing out the expected anomalous non-fickian nature of release. 


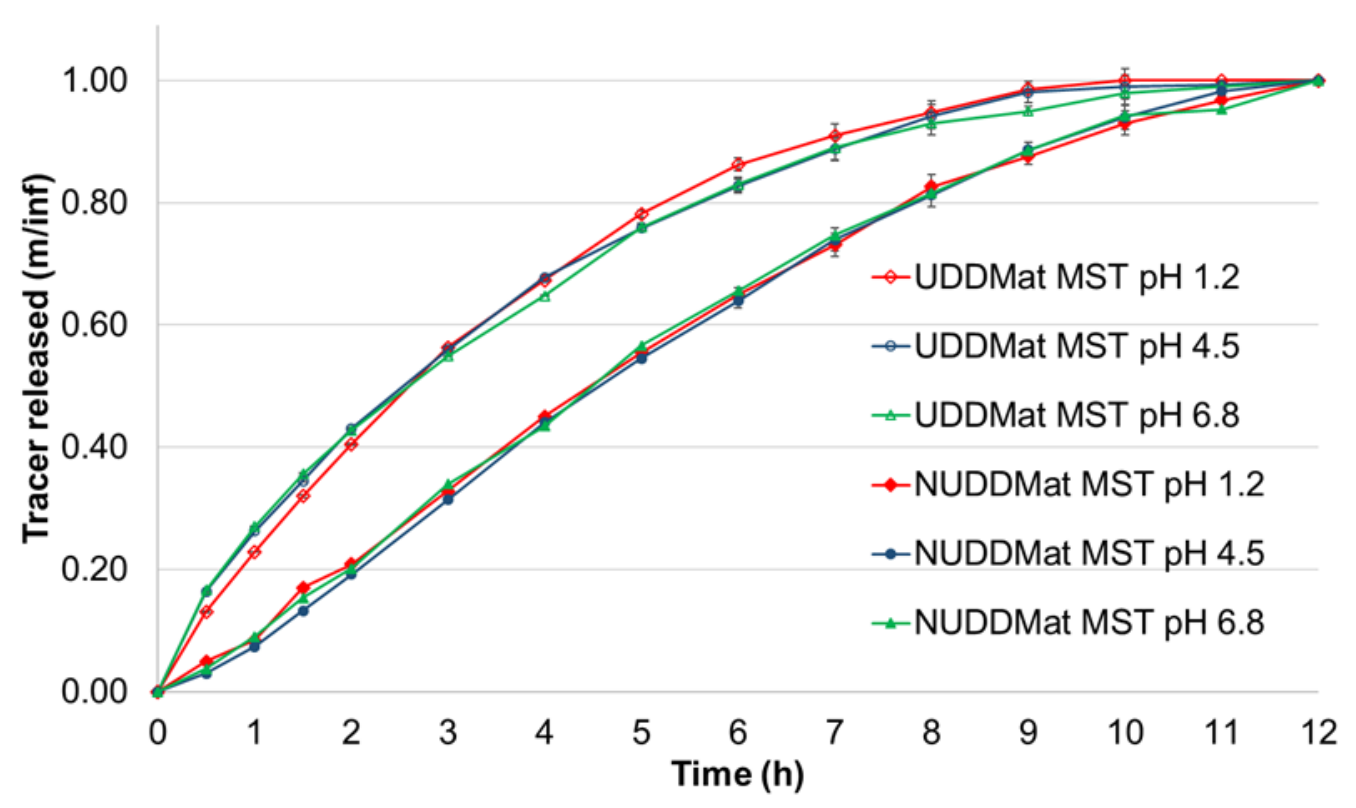

Fig. 8. Release profiles from NUDDMat MST and UDDMat MST systems in pH 1.2, 4.5 and 6.8 fluids.

\section{Conclusions}

According to a formulation design previously demonstrated successful, the NUDDMat hydrophilic matrix system, intended for zero-order release, was manufactured by subsequent deposition onto inert cores of layers having outwards decreasing concentrations, according a staircase profile, of drug tracers having high, medium and low water solubility. Powder layering technique was used, and different mixtures were employed wherein the drug and the release-controlling polymer had percentage compositions set for each of the overlaid layers. The systems showed good physico-technological properties, and the control on the rate of release, irrespective of the solubility characteristics of the tracers, turned out more effective by increasing the number of layers applied. NUDDMat systems provided with 5 layers having $315 \mu \mathrm{m}$ thickness and 420 without drug in the external layer generated in all cases sigmoidal release profiles with an initial low-rate phase. However, by halving the thickness of the $5^{\text {th }}$ layer, linear release of HST and MST was achieved. The lag time had a duration of approximately $4 \mathrm{~h}$ in the NUDDMat SST profile, and only a reduction thereof could be obtained when a halved outer layer was applied. Higher variability of data would in principle support a mainly erosive mechanism of release in the case of systems loaded with the poorly soluble tracer.

425 The pH-independent release behavior of NUDDMat was assessed by testing the MST-containing system as compared with tableted uniform drug distribution system having the same quali-quantitative composition. In particular, similar release profiles were obtained using media having $\mathrm{pH} 1.2,4.5$ and 6.8.

Based on the results obtained, the proposed NUDDMat hydrophilic matrix gradient system was confirmed to be an advantageous delivery platform able to provide zero-order release of drug molecules having different solubility. Further formulation studies, mainly leveraging modulation of the concentration gradient within the matrix and of the thickness of the various layers, need to be faced to broaden the scope of application of NUDDMat technology to poorly water soluble drugs. 


\section{References}

Administration Centre For Drug US Department of Health and Human Services Food and Drug, CDER Evaluation And Research, 1997. Guidance for industry dissolution testing of immediate release solid oral dosage forms, Dissolution Technol.

Bogentoft, C.B., Appelgren, C.H., 1984. United States Patent (19. Bogentoft et al. 54 METHOD FOR PREPARING PREPARATIONS HAVING CONTROLLED. US4289795A.

Cerea, M., Maroni, A., Palugan, L., Bellini, M., Foppoli, A., Melocchi, A., Zema, L., Gazzaniga, A., 2018. Novel hydrophilic matrix system with non-uniform drug distribution for zero-order release kinetics. J. Control. Release 287, 247-256. doi:10.1016/j.jconrel.2018.08.027

Chambers, D., Kelly, G., Limentani, G., Lister, A., Lung, K.R., Warner, E., 2005. Analytical method equivalence - An acceptable analytical practice. Pharm. Technol. 64-80.

Colombo, P., Conte, U., Caramella, C., Gazzaniga, A., La Manna, A., 1985. Compressed polymeric minimatrices for drug release control. J. Control. Release 1, 283-289. doi:10.1016/01683659(85)90004-5

Colombo, P., Conte, U., Gazzaniga, A., Maggi, L., Sangalli, M.E., Peppas, N.A., La Manna, A., 1990. Drug release modulation by physical restrictions of matrix swelling. Int. J. Pharm. 63, 43-48.

Colombo, P., Gazzaniga, A., Caramella, C., Conte, U., La Manna, A., 1987. In vitro programmable zeroorder release drug delivery system. Acta Pharm. Technol. 33, 15-20.

Colorcon, 2000. Using METHOCEL Cellulose Ethers for Controlled Release of Drugs in Hydrophilic Matrix Systems [WWW Document]. 2000. URL http://www.colorcon.com/literature/marketing/mr/Extended Release/METHOCEL/English/hydroph_matrix_broch.pdf (accessed 6.24.16).

Conte, U., Maggi, L., 1996. Modulation of the dissolution profiles from Geomatrix® multi-layer matrix tablets containing drugs of different solubility. Biomaterials. doi:10.1016/0142-9612(96)832844

460 de Haan, P., Lerk, C.F., 1986a. The megaloporous system: a novel principle for zero-order drug delivery I. In vitro and in vivo performance. Int. J. Pharm. 31, 15-24.

de Haan, P., Lerk, C.F., 1986b. The megaloporous system: A novel principle for zero-order drug delivery. II. A model for the mechanism of drug delivery. Int. J. Pharm. doi:10.1016/03785173(86)90010-4

465 Dekyndt, B., Verin, J., Neut, C., Siepmann, F., Siepmann, J., 2015. How to easily provide zero order release of freely soluble drugs from coated pellets. Int. J. Pharm. 478, 31-38.

Del Curto, M.D., Palugan, L., Foppoli, A., Zema, L., Gazzaniga, A., Maroni, A., 2014. Erodible timedependent colon delivery systems with improved efficiency in delaying the onset of drug release. J. Pharm. Sci. 103, 3585-93. doi:10.1002/jps.24150

Deng, G.Y., Xiang, L.Y., Wei, D.H., Liu, J., Yun, G.W., Xu, H., 2007. Tablets with material gradients fabricated by three-dimensional printing. J. Pharm. Sci. doi:10.1002/jps.20864

Durbin, J., Watson, G.S., 1950. Testing for serial correlation in least squares regression. I. Biometrika 38, 159-78. doi:10.1093/biomet/58.1.1

European Pharmacopoeia, 2019. 2.9.34. Bulk density and tapped density of powders, 10.0. ed. European Directorate for Quality of Medicines \& Healthcare, Strasbourg.

Fassihi, R.A., Ritschel, W.A., 1993. Multiple-Layer, Direct-Compression, Controlled-Release System: In Vitro and In Vivo Evaluation. J. Pharm. Sci. 82, 750-754. doi:10.1002/jps.2600820715

Foglio Bonda, A., Rinaldi, M., Segale, L., Palugan, L., Cerea, M., Vecchio, C., Pattarino, F., 2016. Nanonized 
itraconazole powders for extemporary oral suspensions: Role of formulation components studied by a mixture design. Eur. J. Pharm. Sci. 83. doi:10.1016/j.ejps.2015.12.030

Foppoli, A.A., Cerea, M., Melocchi, A., Zema, L., Palugan, L., Maroni, A., Gazzaniga, A., 2019. Evaluation of powder-layering vs. spray-coating techniques in the manufacturing of a swellable/erodible pulsatile delivery system. Drug Dev. Ind. Pharm. submitted.

Foppoli, A.A., Maroni, A., Cerea, M., Zema, L., Gazzaniga, A., 2017. Dry coating of solid dosage forms: an overview of processes and applications. Drug Dev. Ind. Pharm. 43. doi:10.1080/03639045.2017.1355923

Forni, F., Coppi, G., Iannuccelli, V., Vandelli, M.A., 1989. Distribution of drugs in polymers loaded by swelling. J. Pharm. Sci. 25-27.

Gazzaniga, A., Sangalli, M.E., Conte, U., Caramella, C., Colombo, P., La Manna, A., 1993. On the release mechanism from coated swellable minimatrices. Int. J. Pharm. 91, 167-171. doi:10.1016/03785173(93)90336-E

Grassi, M., Zema, L., Sangalli, M.E., Maroni, A., Giordano, F., Gazzaniga, A., 2004. Modeling of drug release from partially coated matrices made of a high viscosity HPMC. Int. J. Pharm. 276, 107-14. doi:10.1016/j.ijpharm.2004.02.016

Hildgen, P., McMullen, J.N.N., 1995. A new gradient matrix: Formulation and characterization. J. Control. Release 34, 263-271. doi:10.1016/0168-3659(95)00014-Y

Huang, K.K., Wang, D.P., Meng, C.L., 2002. Development of extended release dosage forms using nonuniform drug distribution techniques. Drug Dev. Ind. Pharm. 28, 593-599. doi:10.1081/DDC120003455

James Chang, N., Himmelstein, K.J., 1990. Dissolution-diffusion controlled constant-rate release from heterogeneously loaded drug-containing materials. J. Control. Release 12, 201-212. doi:10.1016/0168-3659(90)90101-X

Kim, C. ju, 1995. Compressed Donut-Shaped Tablets with Zero-Order Release Kinetics. Pharm. Res. 12, 1045-1048. doi:10.1023/A:1016218716951

505 Korsmeyer, R.W., Gurny, R., Doelker, E., Buri, P., Peppas, N.A., 1983. Mechanisms of solute release from porous hydrophilic polymers. Int. J. Pharm. 15, 25-35. doi:10.1016/0378-5173(83)90064-9

Lee, P.I., 1986. Initial concentration distribution as a mechanism for regulating drug release from diffusion controlled and surface erosion controlled matrix systems. J. Control. Release 4, 1-7. doi:10.1016/0168-3659(86)90027-1

Lee, P.I., 1984. Novel approach to zero-order drug delivery via immobilized nonuniform drug distribution in glassy hydrogels. J. Pharm. Sci. 73, 1344-1347. doi:10.1002/jps.2600731004

Li, L.C., Tu, Y.H., 1991. Latex film matrix systems with a concentration gradient for controlled drug delivery. Drug Dev. Ind. Pharm. doi:10.3109/03639049109048531

MacLeod, G.S., Fell, J.T., Collett, J.H., 1999. An in vitro investigation into the potential for bimodal drug release from pectin/chitosan/HPMC-coated tablets. Int. J. Pharm. doi:10.1016/S03785173(99)00197-0

Maroni, A., Del Curto, M.D.M.D., Cerea, M., Zema, L., Foppoli, A., Gazzaniga, A., 2013. Polymeric coatings for a multiple-unit pulsatile delivery system: Preliminary study on free and applied films. Int. J. Pharm. 440, 256-263. doi:10.1016/j.ijpharm.2012.05.075

520 Marsac, P.J., Shamblin, S.L., Taylor, L.S., 2006. Theoretical and Practical Approaches for Prediction of Drug-Polymer Miscibility and Solubility. Pharm. Res. 23, 2417-2426. doi:10.1007/s11095-0069063-9

Mazzoni, C., Tentor, F., Antalaki, A., Jacobsen, R.D., Mortensen, J., Slipets, R., Ilchenko, O., Keller, S.S., 
Nielsen, L.H., Boisen, A., 2019. Where Is the Drug? Quantitative 3D Distribution Analyses of Confined Drug-Loaded Polymer Matrices. ACS Biomater. Sci. Eng. doi:10.1021/acsbiomaterials.9b00495

Monkhouse, D., Yoo, J., Sherwood, K.J., Cima, M.J., Bornancini, J.E., Sherwood, J.K., Cima, M.J., Bornancini, E., 2001. Dosage forms exhibiting multi-phasic release kinetics and methods of manufacture thereof. US 6514518 B2.

530 Phaechamud, T., 2008. Variables influencing drug release from layered matrix system comprising hydroxypropyl methylcellulose. AAPS PharmSciTech 9, 668-674. doi:10.1208/s12249-008-90851

Qi, M., Wang, P., Wu, D., 2003. A novel pH- and time-dependent system for colonic drug delivery. Drug Dev. Ind. Pharm. 29, 661-7. doi:10.1081/DDC-120021315

535 Roberts, R.J., Rowe, R.C., 1985. The effect of punch velocity on the compaction of a variety of materials. J. Pharm. Pharmacol. 37, 377-384. doi:10.1111/j.2042-7158.1985.tb03019.x

Sangalli, M.E., Giunchedi, P., Gazzaniga, A., Conte, U., 1993. Erodible perforated coated matrix for extended release of drugs. Int. J. Pharm. 91, 151-156. doi:10.1016/0378-5173(93)90334-C

Sangalli, M.E., Giunchedi, P., Maggi, L., Conte, U., Gazzaniga, A., 1994. Inert monolithic device with a central hole for constant drug release. Eur. J. Pharm. Biopharm.

Sangalli, M.E.M.E., Maroni, A., Zema, L., Cerea, M., Conte, U., Gazzaniga, A., 2003. A study on the release mechanism of drugs from hydrophilic partially coated perforated matrices. Farmaco 58, 971 976. doi:10.1016/S0014-827X(03)00168-X

Scott, D.C., Hollenbeck, R.G., 1991. Design and manufacture of a zero-order sustained-release pellet dosage form through nonuniform drug distribution in a diffusional matrix. Pharm. Res. 8, 156-61.

Siepmann, J., Peppas, N.A., 2001. Modeling of drug release from delivery systems based on hydroxypropyl methylcellulose (HPMC). Adv. Drug Deliv. Rev. 48, 139-57.

Sinclair, G.W., Peppas, N.A., 1984. Analysis of non-fickian transport in polymers using simplified exponential expressions. J. Memb. Sci. 17, 329-331.

Sun, C., 2005. True density of microcrystalline cellulose. J. Pharm. Sci. doi:10.1002/jps.20459

Tabatar, T., Makino, T., Kashihara, T., Hirai, S., Kitamori, N., Toguchi, H., 1992. Stabilization of a new antiulcer drug (Lansoprazole) in the solid dosage forms. Drug Dev. Ind. Pharm. 18, 1437-1447. doi:10.3109/03639049209040850

United States Pharmacopeia Convention, 2017. General Chapters: <1010>Analytical DataInterpretation and Treatment, in: USP 40 The United States Pharmacopeia - NF 35 National Formulary. p. 836.

van Bommel, E.M., Raghoebar, M., Tukker, J.J., 1991. Comparison of in vitro and in vivo release characteristics of acetaminophen from gradient matrix systems. Biopharm. Drug Dispos. 12, 36773.

van Bommel, E.M.G., Fokkens, J.G., Crommelin, D.J.A., 1989. A gradient matrix system as a controlled release device. Release from a slab model system. J. Control. Release 10, 283-292. doi:10.1016/0168-3659(89)90078-3

Van Bommel, E.M.G., Fokkens, J.G., Crommelin, D.J.A., 1990. Production and evaluation of in vitro release characteristics of spherical gradient matrix systems. Acta Pharm. Technol.

565 van der Voet, H., de Haan, P., Doornbos, D.A., 1983. The use of the Durbin-Watson statistic for testing the validity of kinetic models for dissolution. Int. J. Pharm. 14, 291-298. doi:10.1016/03785173(83)90101-1 
Venkateswara Reddy, B., Navaneetha, K., Venkata Ramana Reddy, K., 2014. Process development and optimization for moisture activated dry granulation method for losartan potassium tablets. Int. J. Pharm. Pharm. Sci. 6, 312-317.

Wan, L.S.C., Lai, W.F., 1992. Multilayer drug-coated cores: A system for controlling drug release. Int. J. Pharm. 81, 75-88. doi:10.1016/0378-5173(92)90045-4

Wu, C.-Y., Best, S.M., Bentham, A.C., Hancock, B.C., Bonfield, W., 2005. A simple predictive model for the tensile strength of binary tablets. Eur. J. Pharm. Sci. 25, 331-336. doi:10.1016/j.ejps.2005.03.004

Zema, L., Sangalli, M.E., Maroni, A., Foppoli, A., Bettero, A., Gazzaniga, A., 2010. Active packaging for topical cosmetic/drug products: A hot-melt extruded preservative delivery device. Eur. J. Pharm. Biopharm. doi:10.1016/j.ejpb.2010.03.007

Zoglio, M.A., Carstensen, J.T., 1984. A simple, theoretical approach to zero order controlled drug release from compressed tablets. Int. J. Pharm. Technol. Prod. Manuf. 5, 1-5. 


\section{Acknoledgements}

SEM analysis was carried out at NOLIMITS, an advanced imaging facility established by the Università degli Studi di Milano. The authors wish to thank Nadia Santo for support. 


\section{Captions list}

Fig. 1. Schematic 3D representation of the NUDDMat system. Drug tracer concentration is indicated by color intensity.

Fig. 2. Theoretical concentration patterns for drug tracer, polymer and filler along the radius of the NUDDMat systems.

595 Fig. 3. SEM photomicrographs of HST (a), MST (b) and SST (c) powders.

Fig. 4. Units ranging from core seed to final NUDDMat system containing HST (scale in millimeters).

Fig. 5. SEM photomicrographs of cross-sectioned NUDDMat systems containing HST (a), MST (b) e $\operatorname{SST}(\mathrm{c})$.

Fig. 6. Release profiles from NUDDMat systems containing HST (a), MST (b) and SST (c).

Fig. 7. Release profiles from NUDDMat systems with 5th layer having $157.5 \mu \mathrm{m}$ thickness.

605

Fig. 8. Release profiles from NUDDMat MST and UDDMat MST systems in $\mathrm{pH} 1.2,4.5$ and 6.8 fluids. 